\title{
Outflows from Newborn Multiple Stars
}

\author{
Bo Reipurth \\ Center for Astrophysics and Space Astronomy, \\ University of Colorado, Boulder, CO 80309, USA
}

\begin{abstract}
Analysis of a sample of giant Herbig-Haro flow sources shows that $79 \%-86 \%$ are binaries or higher order multiples. This represents the youngest sample of stars studied so far for binarity. A stellar dynamics jet hypothesis is proposed in which the dynamical decay of triple or multiple systems leads to giant outflow activity. Close triple approaches will cause serious perturbations and probably direct collisions among individual circumstellar disks, with a consequent burst of outflow activity, which can produce giant $\mathrm{HH}$ bow shocks. As one component is ejected, the two remaining stars and their small truncated disks form a closer bound pair with high eccentricity. Gas streams from a circumbinary disk feed the stars and this as well as other dynamical effects cause the binary orbit to shrink. As the stellar components gradually spiral towards each other, accretion and outflow becomes cyclic, modulated on an orbital time scale. The resulting $\mathrm{HH}$ flows can be read as a fossil record of the evolution of orbital motions of the newly formed binary as it shrinks from a typical separation of $100 \mathrm{AU}$ or more to $10 \mathrm{AU}$ or less. After a triple disintegration event, both components (star and close binary) leave their nascent envelope, and while one component becomes visible as a $\mathrm{T}$ Tauri star, the other will be obscured for a while by the envelope and will appear as a bright near-infrared object, thus explaining the socalled IRC binaries which are infrequently found in star forming regions.
\end{abstract}

\section{Introduction}

Outflows from newborn stars can manifest themselves in a variety of ways, including $\mathrm{CO}$ flows observable at millimeter wavelengths, $\mathrm{HH}$ flows seen at optical wavelengths, $\mathrm{H}_{2}$ flows which can be imaged in the infrared, and thermal radio jets detectable at centimeter wavelengths. These appear to be various manifestations of the same underlying phenomenon. Statistical studies of molecular outflows suggest that most or all young stars go through a phase of outflow activity that may last as long as the embedded phase, i.e. about $10^{5} \mathrm{yr}$ (e.g. Bontemps et al. 1996). It is generally accepted that these outflows are the result of accretion flows through circumstellar disks and serve to rid the disks of excess angular momentum. A variety of magnetohydrodynamic models have been proposed by which inflow can be turned to outflow (e.g. Shu et al. 1994, Pudritz \& Norman 1986). 
Given the high frequency of binarity among young stars, it is to be expected that outflows from multiple sources should be abundant. Indeed, a number of quadrupolar outflows have been found in the last decade. Among the better known cases are the flows in L723 (Avery et al. 1990), IRAS 16293-2422 (Mizuno et al. 1990), NGC1333 IRAS2 (Sandell et al. 1994), and IRAS 20050 +2720 (Bachiller et al. 1995), to mention just a few. Similarly, infrared images of $\mathrm{H}_{2}$ flows have revealed flows with four lobes emanating from a single source, as the HH 240 flow in L1634 (Hodapp \& Ladd 1995). Also multiple HH flows from binaries are known, like the double jet from L1551 IRS5 (Fridlund \& Liseau 1998).

Based on an analysis of multiplicity of outflow sources, I here review a new proposal that dynamically unstable multiple systems can lead to outflows and that such outflows may provide a fossil record of orbital motions in disintegrating triple systems. For full details, see Reipurth (2000).

\section{Multiplicity of Giant Outflow Sources}

The most spectacular manifestations of the outflow phenomenon are the giant Herbig-Haro flows, which may stretch over many parsecs (e.g. Reipurth, Bally, Devine 1997). The driving sources are typically deeply embedded Class I sources, and their detection often require infrared or radio continuum techniques. The following giant $\mathrm{HH}$ fow sources have been studied by different groups with a variety of high resolution techniques, which generally allow an assessment of binarity to approximately 0.1-0.2 arcsec: HH 1/2 (from VLA 1), HH 24 (from SSV 63), HH 34 (from HH 34 IRS), HH 47 (from IRAS 08242-5050), HH 83 (from IRAS 05311-0631), HH 111 (from IRAS 05491+0247), HH 154 (from L1551 IRS5), HH 160 (from Z CMa), HH 199 (from IRAS 20582+7724), HH 300 (from IRAS 04329+2436), HH 303 (from L1641-N VLA), HH 355 (from T Tauri), HH 410 (from Haro 6-10), and HH 454 (from L1551-NE).

Of these 14 giant flow sources, 12 show evidence for being at least binaries, and of these 6 are higher-order multiples. Taken at face value, this means that $86 \%$ of these giant outflow sources are binaries and $43 \%$ are higher order multiples, the highest multiplicity frequency ever observed in a sample of young stars. This sample is also by far the youngest sample which has been studied for binarity, with ages of typically less than $10^{5} \mathrm{yr}$, suggesting that dramatic changes in separations or stability of the systems occur in the time interval before the sources become more evolved T Tauri stars, which have smaller binary frequencies (e.g. Reipurth \& Zinnecker 1993, Ghez et al. 1993, Köhler \& Leinert 1998).

It should be noted, however, that the data set is inhomogeneous because of the different observational techniques employed and resulting different resolutions, so that a certain amount of interpretation of the data is inevitably required. Following an evaluation of this, I conclude that the current best evidence suggests that the observed multiplicity frequency of giant $H H$ flow sources is somewhere in the range $79 \%$ to $86 \%$ (see Reipurth 2000 for details). This is consistent with the possibility that all sources of giant HH flows may be binaries or multiples. 


\section{The Properties of Three-Body Interactions}

The chaotic dynamics which characterize triple systems have no analytical solutions and can only be explored through numerical simulations in a statistical manner. A vast literature exists on the subject, which was most recently reviewed by Valtonen \& Mikkolo (1991). The motions of the three bodies while they are bound together can basically be divided into three classes, which interchange at random until the system decays through escape of a member (Figure 1). One class is the interplay, during which the three members perform completely random motions with no periodicity. During interplay two members, often the two most massive, can form a temporary binary, which have frequent two-body encounters, while continuously being perturbed by approaches by the third member, sometimes in the form of a flyby, but occasionally resulting in exchange of binary membership. A second class is the close triple approach, in which all three bodies briefly and more or less simultaneously are brought close together. It is during such events that energy is exchanged between the components. Statistical studies show that close triple approaches are necessary but not sufficient conditions for escape. The third class of motion is the ejection, which will occur following a close triple approach during which a binary is formed that absorbs the potential energy of the third member thereby loosening its ties to the system. At this point the triple transforms into two two-body systems, namely a close binary and an ejected member moving on an approximately elliptic orbit, which eventually brings it back to the close binary. If instead the ejected member moves on a hyperbolic orbit, the ejection becomes an escape. Statistical studies show that it is usually, but not always, the lightest member that is ejected; the escape probability scales approximately as the inverse third power of the mass. Although eventually most non-hierarchical triple systems disintegrate in an escape, this is clearly not always the case, as the presence of stable hierarchical triple systems among main sequence stars demonstrates (e.g. Tokovinin 1997). Finally, it is important to note that numerous studies have shown that the close binaries which form as a result of the escape of a third member usually are highly eccentric, with eccentricities exceeding 0.9 not being unusual (e.g. Valtonen \& Mikkola 1991).

The numerical simulations of Sterzik \& Durisen $(1995,1998)$ and Armitage \& Clarke (1997) show that typical escape speeds for low-mass stars are 3-4 km $\mathrm{s}^{-1}$, but with a non-neglible tail of the distribution out to $20 \mathrm{~km} \mathrm{~s}^{-1}$ and beyond. It is not possible to define the precise lifetime of a triple system, because it can be shown that the decay of a large number of triple systems occurs randomly. However, approximately within a hundred crossing times about $95 \%$ of the triple systems have decayed (Sterzik \& Durisen 1995). For parameters which are likely to hold among newly born multiple stars, decay times are of the order of several times $10^{4} \mathrm{yr}$.

When applying the above considerations to star forming regions, it is important to recall that young stars cannot be treated as point sources moving in a vacuum. Rather, the members of newborn triple systems have disks and move inside an infalling envelope, so gas dynamical forces become important. It is particularly interesting to consider how circumstellar material is re-distributed during a close triple approach. Figure 1 gives a schematic illustration of the three types of motion in a triple system. During interplay the stellar seeds pre- 


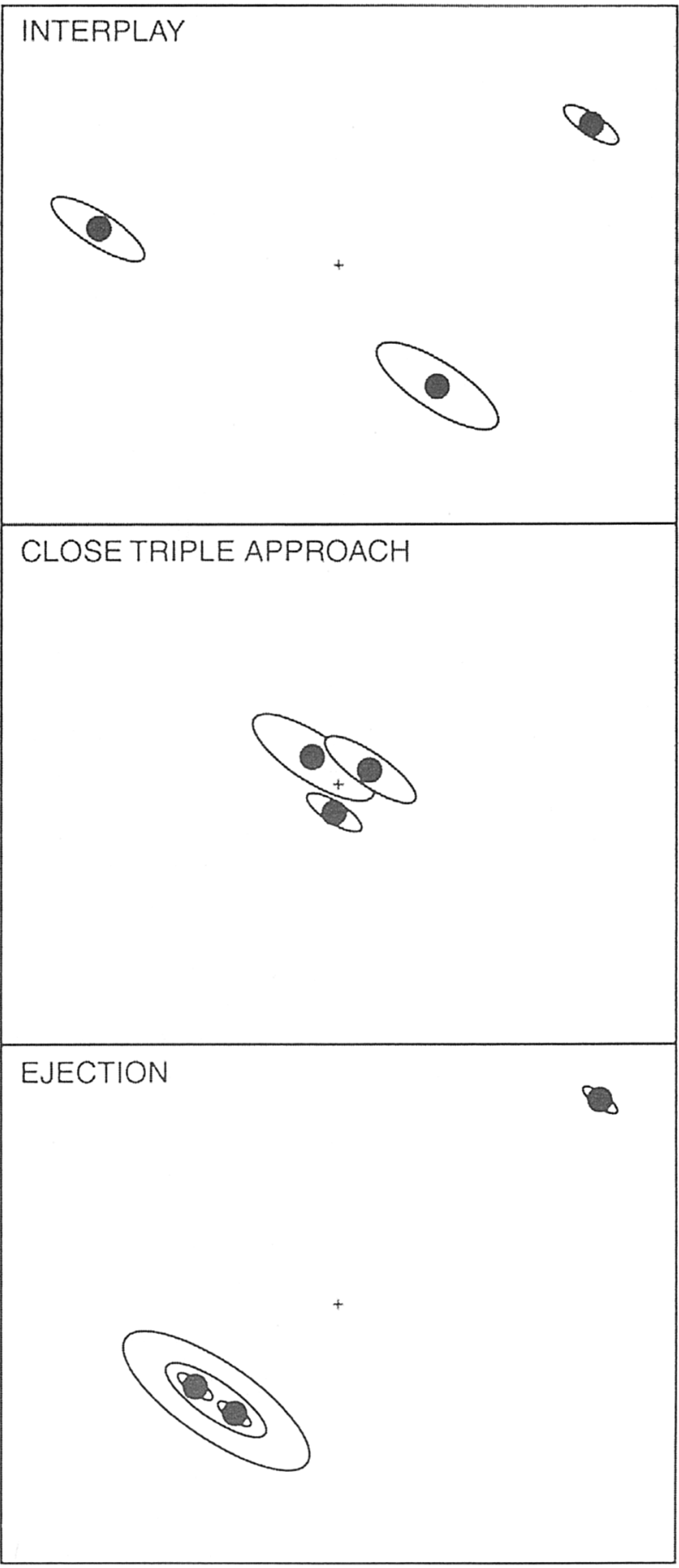

Figure 1. A schematic presentation of the three phases in the dynamical break-up of a young non-hierarchical triple system. 
sumably develop substantial circumstellar disks, from which they build up their stellar masses. When a close triple approach occurs, the stars themselves are affected only insofar that their energy and angular momentum may change, but their disks suffer major tidal distortions and perhaps even direct collisions. As a result the circumstellar disks are seriously truncated, but much of the material culled from the three circumstellar disks may find a new home in a circumbinary disk around the newly formed binary.

\section{Disk-Disk Interactions and Giant HH Bow Shocks}

Do the chaotic interactions in newborn multiple systems lead to giant $\mathrm{HH}$ flows? It could be argued that there is no causal relation between multiplicity and outflow activity, and that the observed high multiplicity frequency among giant $\mathrm{HH}$ flow sources merely follows because triple systems are likely to decay on time scales of several times $10^{4} \mathrm{yr}$. Since the main outflow activity of young stars occur within their first $10^{5} \mathrm{yr}$, then the two are almost bound to coincide. Far more detailed studies than are available at the time of writing are required to prove a causal relation.

Instead, based on the available observational evidence and on the current understanding of star formation processes, I here postulate that the massive outflow activity which result in the giant jets is a direct consequence of the disintegration processes of newborn triple systems. Whether any other processes in the formation of single stars can also lead to giant $\mathrm{HH}$ flows is neither disputed nor further considered here. In the following I examine in qualitative terms a plausible scenario.

Let us envisage a prototriple system, which is in the process of forming, probably as the result of fragmentation in a cloud core, and which is accreting gas from an infalling envelope (e.g. Bodenheimer et al. 2000). As long as the stellar embryos still have small masses and infall is massive, gravitational interactions between the stellar masses is presumably limited. However, as the stellar masses grow, the interplay phase of the triple system begins. At this early stage still dominated by massive infall, individual circumstellar disks are likely to be relatively transient features, which are built up, perturbed or even destroyed, and then re-assembled because of the two- and three-body interactions continuously taking place (e.g. Hall, Clarke, Pringle 1996). While perturbation is fast in the shortlived periastron passages, new versions of the circumstellar disks re-assemble during the much longer periods around apastron.

Really serious disturbances and truncations of the circumstellar disks occur when the close triple approaches take place, leading to significant outflow activity. The issue is how disk perturbations from a companion, which by the nature of the process has most effect on the outer parts of a disk, can affect the innermost parts of the disk, from where the jets are launched. As tidally generated spiral waves move in through a disk, they will decay because shocks provide wave damping (e.g. Spruit 1987) and turbulent viscosity may also act as a source of dissipation. Only for the most massive disks with masses of more than, say, $0.1 \mathrm{M}_{\odot}$ are external perturbations likely to affect the innermost disk regions. But, as noted by Larson (2001), even if tidal waves are strongly damped 
at some radius, inflowing matter may pile up there and might eventually cause a gravitational instability leading to further inflow of matter.

However, a simpler mechanism may operate. In close triple approaches, the disks may actually physically crash into each other and exchange mass. Significant mass capture and mass loading of the small inner surviving disks may subsequently take place by material stripped-off from the outer disks, bypassing the need for transmitting a disturbance. Such episodes will bring the study of PMS binaries closer to that of cataclysmic variables and other mass-exchange and accretion powered binary phenomena (e.g. Sahai \& Nyman 2000). For all of such systems it is increasingly well established, both observationally and theoretically, that increased accretion in a circumstellar disk is related to outflow activity, and the sudden onset of massive accretion in a close triple approach should initiate a violent burst of outflow activity.

As vividly demonstrated in the numerical SPH simulations of Artymowicz \& Lubow $(1994,1996)$, binaries will form gaps in their circumbinary disks through which gas streams will flow. The less massive companion $b$ drags out a stream of gas when it is near apastron, and especially for high eccentricity binaries, such as will result from a triple disintegration event, the simulations show that the gas streams vary strongly with time. The inflowing gas will add to the individual small circumstellar disks, and may pile up until the disk becomes gravitationally unstable and enhanced mass accretion occurs. This will happen typically once per orbital period of the close binary. The MHD process which launches the jet is then fed on a semi-regular basis. The amount of accepted material may vary, and the periodicity may be perturbed, because of inhomogeneities in the circumbinary disk, which gravitationally perturb the binary. It is also possible that the very high eccentricity of a binary formed after a close triple approach will lead to severe disk interactions, including direct collisions, at periastron passages. Such interactions may be repetitive as the stars continue to accumulate material during each orbit, only to see their disks get truncated at periastron. A full understanding of disk-disk interactions await extensive numerical studies, which are only beginning to appear (e.g. Watkins et al. 1998).

Whatever the precise mechanism will turn out to be, it seems that companion-induced accretion could cause outflow activity that is modulated on a timescale corresponding to the quasi-periodic orbital motion. In this context it is noteworthy that variable mass accretion regulated by the orbit of a young binary has already been documented, although on a very much smaller scale, in the spectroscopic PMS binary DQ Tau (Mathieu et al. 1997).

\section{Orbital Decay and Highly Collimated Jets}

Giant HH flows are characterized by ejecta which are launched on several different time scales. Firstly, when multiple bow shocks are seen in giant flows, their separations suggest that they are typically launched every one or two thousand years.

A second time scale is defined by the widely spaced knots found much closer to the sources in numerous HH flows. Although these flows have well defined outflow axes, they are certainly not what is generally understood to be finely 
collimated jets. Many HH flows show these kinds of widely spaced HH knots, with characteristic time scales of many hundred years.

A third time scale is defined by the knots in the very finely collimated jets which are occasionally found to emanate from young stars (Figure 2). The typical time interval of ejection is around 20-30 yr.

Altogether, we find time scales of a few thousand years for giant bow shocks, of many hundred years for smaller, widely spaced HH knots, and of decades for the best collimated, bona fide jets. With reference to the previous sections, I interpret these three different time intervals in terms of the disintegration of young triple systems. In this picture, the longest timescale is associated with close triple approaches, during which massive outflow events take place. The two shorter time scales are linked to the period subsequent to ejection of one or more members, at which time the two remaining stars have formed a closer highly eccentric binary in which individual components feed from the circumbinary disk and produce small outflow bursts on an orbital time scale.

Assuming that the stellar masses add up to a total of $2 \mathrm{M}_{\odot}$, a time interval between close triple approaches of, say, $2000 \mathrm{yr}$ corresponds to a semimajor axis of $200 \mathrm{AU}$ if we were dealing with an ordinary binary system. For a nonhierarchical triple system it presumably only gives an indication of the volume of space within which the three stars move around. A time interval of, say, $500 \mathrm{yr}$ between $\mathrm{HH}$ objects in a large-scale aligned flow corresponds to a semimajor axis of about 80 AU. Similarly, the time intervals of about 20-30 yr between the small knots in a collimated jet corresponds to a semimajor axis of 9-12 AU between the stellar components.

Altogether, we may be able to read the structure of giant $H H$ flows as a fossil record of the evolution of orbital motions in disintegrating triple systems.

The numerical simulations of Sterzik \& Durisen (1998) show that the typical semi-major axis of a binary formed in a triple disintegration event is about 5 times smaller than the original few-body system characteristic size. A typical change would be from, say, 150-250 AU to around 30-50 AU. This is still 35 times larger than the size suggested by ejection intervals of knots in highly collimated jets. So if jets are formed as a companion-induced modulation of the outflow, further orbital decay must occur. There are various ways in which the binary orbit could decay:

a) The orbital elements of the newly formed binary will be affected by the presence of the also newly formed circumbinary disk. Artymowicz et al. (1991) show that energy and angular momentum is lost from the binary to the circumbinary disk through the action of resonances, and the effect on the orbit is to reduce binary separation and increase eccentricity.

b) Dynamical friction will affect the energy and angular momentum evolution of the binary orbit. Dissipation leads to a shrinking orbit and increased eccentricity, but on time scales that are comparable to or longer than the embedded phase (Gorti \& Bhatt 1996), so it is not immediately clear how large an effect this causes. However, the presence of disks around the stars may increase the density asymmetry in the wake of the stars, increasing the importance of this effect.

c) When a star is ejected in a close triple approach it will not always escape, but can enter into a long-period orbit with the binary. The non-hierarchical 
system has then been converted into a close and a wide binary. Each time the third member is at periastron, small amounts of energy and angular momentum may be exchanged between the three stars, leading to further shrinkage of the small binary orbit, and an increase in semi-major axis of the larger binary orbit.

d) Another way to further reduce the orbit of the newly formed binary would be if the system initially is quadruple or quintuple, and undergoes multiple disintegration events. In such cases, binding energy may be transferred several times among the stellar components. A cascade of decays may form a very tight binary already at such an early stage that it will still be surrounded by significant circumbinary material.

e) Finally, if the stars are born in a clustered environment, close encounters with other young stars will cause perturbations of a binary orbit, which on average tend to make the binary more tightly bound (Heggie 1975, Larson 2001).

The relative importance of these various mechanisms needs to be studied in detail. But it appears that the formation of a binary is only the beginning of a dynamical phase during which the mass reservoir for feeding the stars shifts from the increasingly truncated individual circumstellar disks to the circumbinary disk, and during the same period that jets are formed. Orbital motion of the binary will create gas streams that will feed the small circumstellar disks from the larger circumbinary disk. Instead of the quasi-steady accretion predicted through the disk of a single star, the gas streams create a strong modulation in the accretion rate, and therefore in the outflow activity, on an orbital or semi-orbital time scale. The stellar dynamics jet hypothesis predicts that the spectacular, highly collimated $H H$ jets occasionally observed in star forming regions witness the rather brief final phase of dynamical interaction when a wider pair of young stars with a separation of 100 AU or more has turned into a closer binary with a separation of 10 AU or less.

\section{Consequences for Binary PMS Evolution}

A small fraction of $\mathrm{T}$ Tauri stars are known to have infrared companions, the most famous being T Tauri, Z CMa and Haro 6-10, all of which are sources of giant $\mathrm{HH}$ flows. Such systems are known as IRC binaries, and about a dozen have been identified so far (e.g. Koresko, Herbst, Leinert 1997). These binaries are puzzling, because they pair together stars which appear to be in very different evolutionary stages, the optical component being a more typical $\mathrm{T}$ Tauri star, and the infrared companion being a class I or extreme Class II source. Several models have been forwarded to explain such systems, among them the idea that a special viewing geometry permits one circumstellar disk to obscure the other star (e.g. Solf \& Böhm 1999) or the idea that the IR companion is undergoing an episode of enhanced accretion (e.g. Koresko, Herbst, Leinert 1997). The stellar dynamics jet hypothesis discussed above offers a natural explanation of the IRC phenomenon, in which IRC binaries represent a brief transition period in the breakup or re-organization of a triple system.

When a young non-hierarchical triple system breaks up into an ejected member and a tight binary, we will observe three stages in this event: In Stage A, all components are inside the flattened envelope, either because 1) the break-up has not yet occurred or 2) it has happened so recently that all components are 


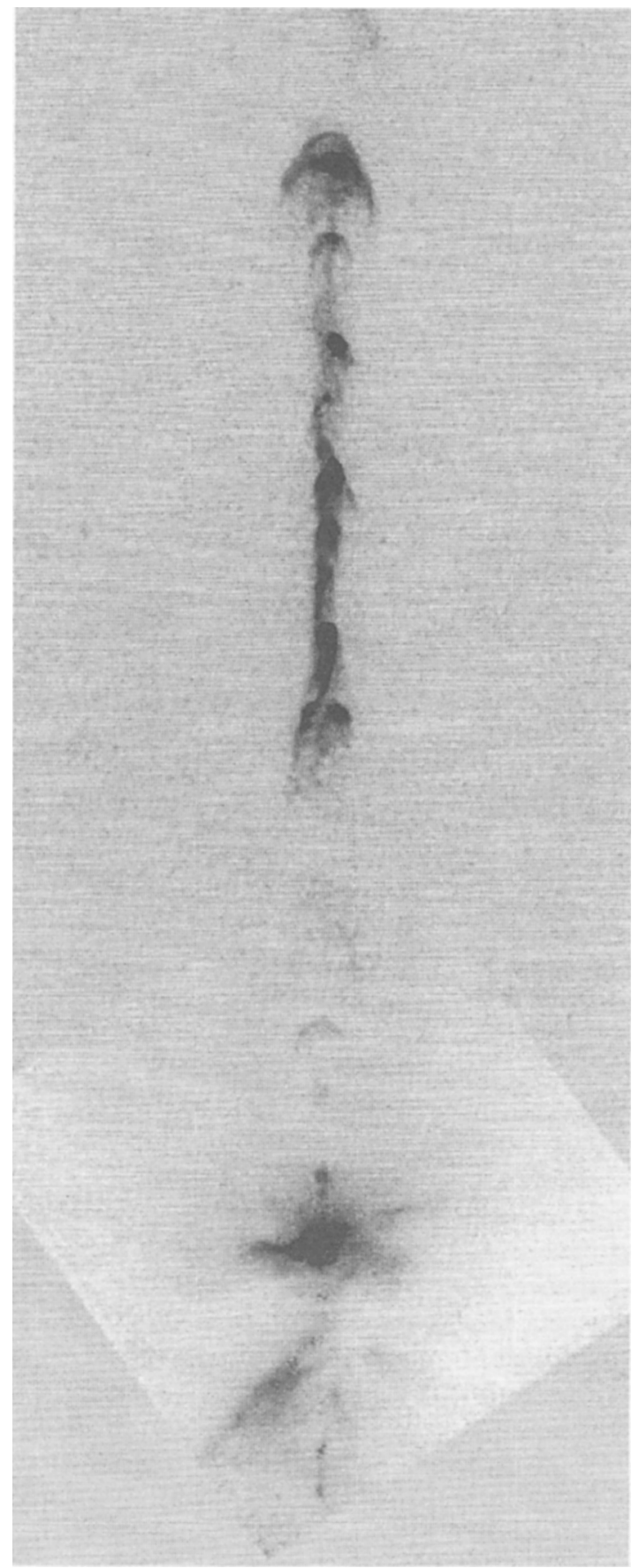

Figure 2. The HH 111 jet emanates from an embedded young triple system. The upper part of the mosaic is an optical HST image, and the lower part a near-infrared HST image. From Reipurth et al. (1999). 
still close to their center of mass within the envelope, or 3) the trajectories of the members of the disintegrating triple pass near the more extensive plane of the flattened envelope. In this stage, the system is observed as a class 0 or (more likely) a class I embedded source. In Stage B, one component has moved outside the envelope, while the other is still within or behind the envelope. This situation will be observed as an IRC binary, with a relatively bright visual component and a nearby heavily extincted infrared companion. In Stage $C$, all components have left the envelope, which meanwhile has cooled off and is in the process of dispersing.

If the ejected star does not escape the system, then the full evolution discussed above does not take place, and the system remains in Stage A, possibly with cyclic excursions into Stage B, and the chaotic motion of the nonhierarchical triple is replaced by the somewhat more ordered motion of a hierarchical triple interacting with the envelope. Since the IRC stage in the bound case might be repeated until the flattened envelope has dispersed, whereas it occurs only once in the unbound case, it is possible that bound IRC binaries are more common than unbound ones.

The stellar dynamics jet hypothesis as related to the IRC phenomenon has a number of observational consequences:

1. All IRC binaries should, without exception, be triple (or higher order multiple) systems.

2. Conservation of momentum in the disintegrating triple system dictates that the velocity of a component (single star $c$ or close binary $a+b$ ) is inversely proportional to its mass. It will therefore traverse a given distance in a time proportional to its mass: If the ejected member has a mass only a quarter of the combined mass of the other two stars it will move four times further in a given interval. If we assume that the lightest member of the system is ejected, it follows that, by definition, the mass of the ejected member $c$ is less than half of the total binary $a+b$ mass. It will therefore spend less than half as long traversing the extent of the envelope as the binary. As component $c$ has the same chance as components $a+b$ of being obscured by the envelope along our line of sight, it follows that systems with component $c$ being obscured stay in the IRC phase less than half as long as if components $a+b$ are the obscured ones. Statistically, we should therefore expect that at least 67\% (corresponding to $m(a)+m(b)=2 m(c)$ ) ranging up to, say, $80 \%$ (corresponding to $m(a)+m(b)$ $=4 \mathrm{~m}(\mathrm{c})$ ) of IRC systems should have a close binary as infrared companion.

3. Using the same argument, for $67 \%$ to $80 \%$ of IRC systems, the infrared companion should have the larger bolometric luminosity, since it is more likely to consist of two stars, which are also more massive. However, this is only true for steady accretion rates, and it is especially likely that IRC systems will have increased and irregular accretion, which may strongly affect the luminosity of the components at any given time. Observations indicate that most infrared companions have the higher luminosity (Koresko et al. 1997).

4. The lifetime of the IRC phenomenon should be short, corresponding to the traversal time behind the flattened envelope. Assuming a characteristic extent of the flattened high-extinction envelope of, say, $1000 \mathrm{AU}$, and a typical motion of the binary (which is more likely to be the IRC component) of about 1 $\mathrm{km} \mathrm{sec}^{-1}$, we arrive at a traversal time of about $5000 \mathrm{yr}$. The fraction of young 
stars with an IRC companion will depend on the age limits set on the sample. If we limit ourselves to ages of less than a few hundred thousand years, only a few percent of young stars should have an IRC companion.

5. The components of an IRC binary should be variable. Given the perturbations which their circumstellar disks have experienced, accretion should be irregular. Additionally, all inhomogeneities in the flattened envelope along the line-of-sight to the infrared companion will be directly observable as variable extinction as the star is moving out behind the envelope. For an assumed projected motion of $1 \mathrm{~km} \mathrm{sec}^{-1}$, the infrared companion will traverse $0.2 \mathrm{AU}$ per year. It appears highly likely that the envelope is not homogeneous on such large scales, and so IRC companions should, in addition to variable accretion, show significant changes in extinction on time scales of a year or shorter.

6. For the reasons outlined in the previous point, the visible T Tauri stars in IRC binaries should show spectroscopic characteristics of strong accretion.

7. All IRC binaries without exception should be found intimately associated with molecular clouds, since the break-up of a triple system statistically should occur on time scales of less than the time scale of the embedded phase.

8. A dispersing envelope of gas and dust should be present in all IRC binaries. In principle, for the more evolved systems with larger separations, a displacement between the stars and the envelope gas might be observable with high resolution millimeter interferometers. This should be true also for the extinction of the dust in the envelope. However, the dust in the envelope will cool on very short timescales, and so the peak of the dust emission will always remain around the star.

The above statements will be true irrespective of whether the IRC binary is the result of an escape or is cyclic because the third member remains bound.

\section{The Case of the HH 111 Flow}

The HH 111 jet is finely collimated and forms part of a giant bipolar HH flows which stretches across $7.7 \mathrm{pc}$ in Orion. The jet emanates from a $25 \mathrm{~L}_{\odot}$ embedded source. Figure 2 shows a composite of optical HST images in $\mathrm{H} \alpha$ plus [SII] emission together with near-infrared images obtained with NICMOS revealing the obscured source region. The near-infrared images have detected the $\mathrm{HH}$ 111 source as a very reddened source. Additional VLA maps have revealed two thermal radio jets emanating from the $\mathrm{HH} 111$ source at right angles to each other, showing that the source itself is a close binary with a projected separation of less than $0.1 \operatorname{arcsec}(50 \mathrm{AU})$. The NICMOS images identified another star 3 $\operatorname{arcsec}(1400 \mathrm{AU})$ away, which was also detected in the radio continuum (and is called VLA 2) and is therefore also very young. The HH 111 binary source is heavily reddened, while the third star is not, and so VLA 1 and 2 form an IRC binary. This has been interpreted in terms of the break-up of a non-hierarchical triple system, in which two components (VLA 1) now form a tight binary, and the third (VLA 2) is ejected. The high reddening of VLA 1 is due to its current location behind the large flattened envelope, while VLA 2 is ejected from its obscuring environment. The $\mathrm{HH} 111$ jet has a space velocity of about $300 \mathrm{~km}$ $\mathrm{s}^{-1}$, suggesting intervals between ejections of $15 \mathrm{yr}$, which suggests a semi-major axis of about 6-8 AU (0.01 arcsec). For full details, see Reipurth et al. (1999). 


\section{References}

Armitage, P. J., Clarke, C. J. 1997, MNRAS, 285, 540

Artymowicz, P., Lubow, S. H. 1994, ApJ, 421, 651

Artymowicz, P., Lubow, S. H. 1996, ApJ, 467, L77

Artymowicz, P., Clarke, C. J., Lubow, S. H., Pringle, J. E. 1991, ApJ, 370, L35

Avery, L. W., Hayashi, S. S., White, G. J. 1990, ApJ, 357, 524

Bachiller, R., Fuente, A., Tafalla, M. 1995, ApJ, 445, L51

Bodenheimer, P., Burkert, A., Klein, R. I., Boss, A. P. 2000, in Protostars and Planets IV, ed. V. Mannings, A. Boss, S. Russell, Univ. of Arizona Press, 675

Bontemps, S., André, P., Terebey, S., Cabrit, S. 1996, A\&A, 311, 858

Fridlund, C. V. M., Liseau, R. 1998, ApJ, 499, L75

Ghez, A. M., Neugebauer, G., Matthews, K. 1993, AJ, 106, 2005

Gorti, U., Bhatt, H. C. 1996, MNRAS, 283, 566

Hall, S. M., Clarke, C. J., Pringle, J. E. 1996, MNRAS, 278, 303

Heggie, D. C. 1975 , MNRAS, 173, 729

Hodapp, K.-W., Ladd, E. F. 1995, ApJ, 453, 715

Koresko, C. D., Herbst, T. M., Leinert, Ch. 1997, ApJ, 480, 741

Köhler, R. Leinert, C. 1998, A\&A, 331, 977

Larson, R. B. 2001, in IAU Symposium No. 200 on The Formation of Binary Stars, ed. H. Zinnecker \& R. D. Mathieu, ASP, this volume

Mathieu, R. D., Stassun, K., Basri, G., Jensen, E. L. N., Johns-Krull, C. M., Valenti, J. A., Hartmann, L. W. 1997, AJ, 113, 1841

Mizuno, A., Fukui, Y., Iwata, T., Nozawa, S., Takano, T. 1990, ApJ, 356, 184

Pudritz, R. E., Norman, C. A. 1986, ApJ, 301, 571

Reipurth, B. 2000, AJ, in press

Reipurth, B., Zinnecker, H. 1993, A\&A, 278, 81

Reipurth, B., Bally, J., Devine, D. 1997, AJ, 114, 2708

Reipurth, B., Yu, K. C., Rodríguez, L. F., Heathcote, S., Bally, J. 1999, A\&A, $352, \mathrm{~L} 86$

Sahai, R., Nyman, L. A. 2000, ApJ, 537, L145

Sandell, G., Knee, L., Aspin, C., Robson, I., Russell, A. 1994, A\&A, 285, L1

Shu, F. H., Najita, J., Ostriker, E., Wilkin, F., Ruden, S., Lizano, S. 1994, ApJ, 429,781

Spruit, H. 1987, A\&A, 184, 173

Sterzik, M. F., Durisen, R. H. 1995, A\&A, 304, L9

Sterzik, M. F., Durisen, R. H. 1998, A\&A, 339, 95

Tokovinin, A. A. 1997, Astronomy Letters, 23, 727

Valtonen, M., Mikkola, S. 1991, ARAA, 29, 9

Watkins, S. J., Bhattal, A. S., Boffin, H. M. J., Francis, N., Whitworth, A. P. 1998, MNRAS, 300, 1205 